\title{
Characterization of VvmiR166s-Targets Modules and their Interaction Mechanism in Modulation of GA-Induced Grape Seedless Berry Process
}

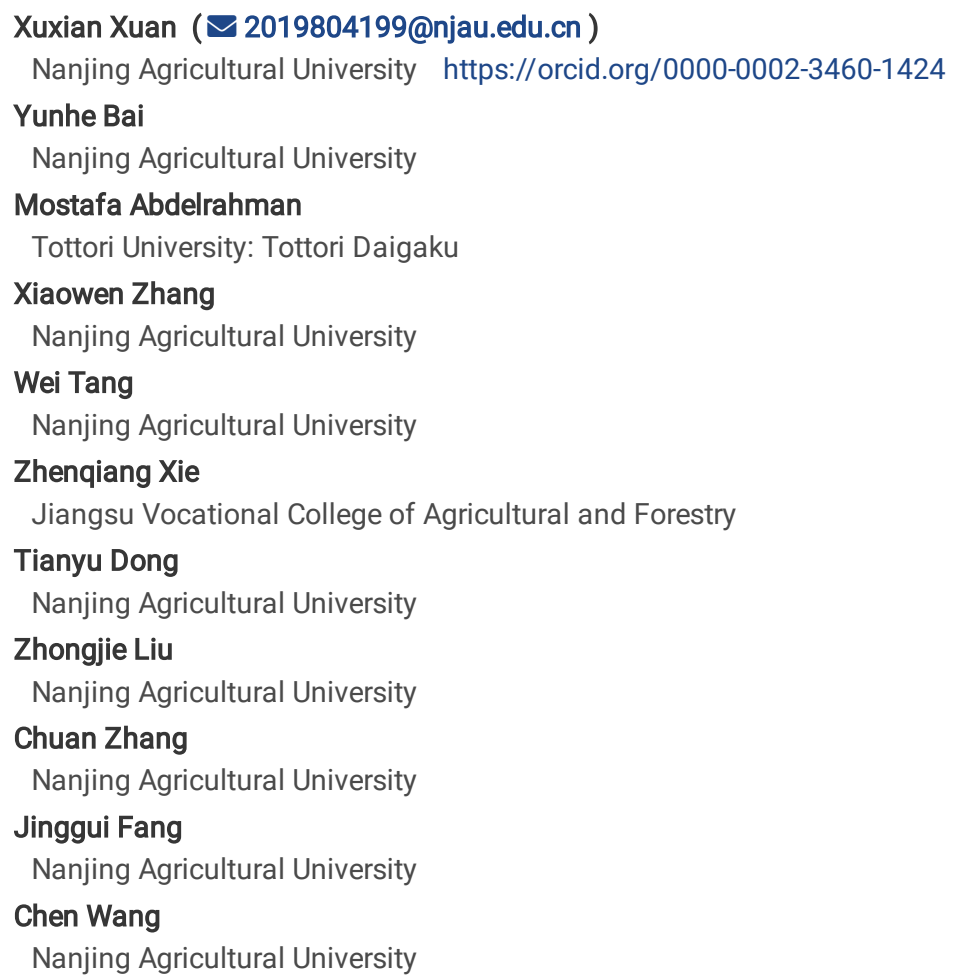




\section{Abstract}

GA (Gibberellin) is one key hormone of inducing grape seedless berries. VvmiR166s were revealed to be the important regulator of responding to GA in grapes, but their roles remain unclear yet. Here, the precise sequences of VvmiR166s and their potential targets $V V R E V, V v H B 15$ and $V V H O X 32$, were determined in grape cv. 'Rosario Bianco'. The cleavage interactions and variations of VvmiR166s:( $V v H B 15, V v H O X 32, V V R E V)$ were validated by RLMRACE and PPM-RACE. Promoters cis-elements analysis supported that VVMIR166s and targets containing similar GA-related cis-elements, might play roles in responding to GA.Subsequently, spatio-temporal expression modes analysis of VVMIR166s and their targets in grape berries suggest that, almost all VVMIR166s possessed the lowest expression level at 46DAF (stone hardening stage) in seeds and peels under GA treatment except for VVMIR166h. Interestingly, their target gene $V v H B 15$ exhibited the opposite expression, indicating that GA promoted the negative regulatory roles of VvmiR166s on $V v H B 15$. Subsequently, over-expressions of $V V M I R 166 b / h$ in Tobaccos, decreased the expression of its target $V v H B 15$ and obviously strengthened some key genes in lignin synthesis pathway, while over-expression of $V V H B 15$ showed the reverse regulatory modes, confirming that VvmiR166b/h might be amongst main factors of regulating lignin synthesis by mediating the expression of $V v H B 15$. The results was deduced a key signal pathway of "GA-VvmiR166b/h-VvHB15- lignin synthesis genes". Our findings revealed a novel insight into the roles of VvmiR166s response to GA in modulating the lignin synthesis of grape seed-stone development, which is essential for the molecular breeding of seedless grape berry.

\section{Key Message}

VvMIR166b/h-VvHB15 modules have been identified in grapes responding to GA, and over-expressions of VvMIR166b/h in Tobaccos strengthened some key genes in lignin synthesis pathway.

\section{Introduction}

Grapevine (Vitis Vinifera L.) is an important economical fruit crop cultivated for the production of table berries, wine, juice and raisins worldwide. Berries with/without seeds directly affect the quality of these products above, and especially, seedlessness is the much important quality trait of table berries and raisins. So far, less seedless grape cultivars are difficult to meet the needs in grape production, and thus GA were widely used to induce seedless grape berries in production (Zhang et al. 2019; Wang et al. 2018; Wang et al. 2020). However, the mechanism of GA-induced seedless grape berries is unclear yet. A deep understanding of this mechanism will be helpful in guiding seedless berry production to improve berry quality.

MicroRNAs (miRNAs) are small single-stranded non-coding RNAs with 20-24nucleotides (nts) in length, which regulate the target gene expression at the post-transcriptional level by degrading and/or translation repression(Wang and Qi 2018; Abdelrahman et al. 2018; Song et al. 2019). Currently, a large number of plant miRNA families with their predominant regulatory role in plant development and reproduction, as well as stress responses have been identified (Leung and Sharp 2010; Kinoshita et al. 2012; Ferdous et al. 2015;Wang et al. 2019). For example, miR156 family regulated SQUAMOSApromoter binding-like (SPL) genes to control the developmental and flowering timing in Arabidopsis (Wang et al. 2009 ), and transgenic tomato (Solanumlycopersicum) plants over-expressing SImiR156 had a semi-dwarf phenotypic character with numerous leaves and longer vegetative phase(Zhang et al. 2011). Similarly, miR172 family was known for the regulation of the juvenile to adult developmental phase through repressing APETALA2 (AP2) transcriptional factors (TFs) in Arabidopsis (Chen 2004; Wu et al. 2009), and over-expression of miR172 in Jatropha (Jatropha curcas) reduced phloem and pith development, enhanced xylem development, resulted in early flowering and altered leaf morphology(Tang 2018). Although the investigation of the regulatory roles of miRNAs uncovered a new avenue in plant biology, the roles of many miRNA families remain to be discovered.

MiR166 is a highly conserved multi-gene family of miRNAs and 262 miR166members have been identified in 45 plant species according to the miRBase database (http://www.mirbase.org), indicating that miR166s possessed the high degree of functional conservation(Li et al. 2017 ; Singh et al. 2017). However, spatio-temporal expression patterns of miR166 genes suggested that functional specialization also existed in miR166 family members(Miyashima et al. 2013; Barik et al. 2014). For instance, miR166a and miR166b were found to be specifically expressed during the postembryonic meristem and root endodermis development in Arabidopsis (Miyashima et al. 2011). Likewise, miR166a, miR166b and miR166g were observed to be highly expressed during early embryogenesis, whereas other miR166 members was not detected in Arabidopsis (Miyashima et al. 2013). Thus, the spatio-temporal expression of VvmiR166s might be tissue-specific at particular stages during plant development, and dressing this diversification is an important step towards understanding their fine-tuned regulatory roles in plant development.

Previous studies reported that miR166 targets five members of the class III homeo domain-leucine zipper (HD-ZIP III) TF genes, including REVOLUTA(REV), PHABULOSA (PHB), PHAVOLUTA (PHV), ARABIDOPSIS THALIANA HOMEOBOX 8 (ATHB8) and ATHB15 in a broad range of plant species(Singh et al. 2017; Williams et al. 2005; Kim et al. 2008; Carlsbecker et al. 2010). In soybean (Glycine max), analyzing the cis-elements of promoters of 26 MIR166 members showed that MIR166s contained highly-conserved binding site of HD-ZIP III family, implying that a possible HD-ZIPIIImediated feedback regulatory loop for miR166 expression existed in soybean(Li et al. 2017). Similarly, Arabidopsis plants over-expressing MIR166a exhibited reduced transcript levels of $A t P H B, A t P H V$ and $A t H B 15$, and altered floral and leaf morphology, including stunted growth, disrupted floral structure, enlarged shoot apical meristem and expanded xylem tissue, indicating that miR166 family is important to vascular and floral development (Kim et al. 2008; Floyd et al. 2006; Ko et al. 2006; Wenkel et al. 2007; Jung et al. 2007). In addition, over-expressing AtHB8 in Arabidopsis plants enhanced cell lignification and xylem formation, while over-expressing AtHB15 repressed the accumulation of lignified tissue in transgetic Arabidopsis, 
indicating that $A t H B 15$ and $A t H B 8$ might have antagonistic roles during vascular development (Kim et al. 2005; Côté et al. 2010; Itoh et al. 2008; Ohashiito et al. 2005).

Spatio-temporal expression of miRNAs in different species and their targets depends on the cis-elements, including hormone response elements, in their respective promoters(Wang et al. 2018). GA is one of the most important phytohormones, and has been reported to function in hypocotyl elongation, seed germination, leaf development and flower induction in wide range of plant species(Wang et al. 2019;Wang et al. 2018; Zhang et al. 2019). In grape (Vitis vinifera), it is well known that GA plays a significant role in grape flower development and seedless berry induction and expansion, which are important traits for the production of highly quality grape (Wang et al. 2019; Wang et al. 2012; Acheampong et al. 2017). However, the molecular mechanisms of miR166-HD-Zip III interactions remain unclear during the complex developmental process of grape berries and seeds under GA treatment. Based on the above studies, we hypothesized that both GA and miR166s as well as HD-Zip III family might participate in the mediation of seed developmental process in grape. Thus, we first isolated and determined the precise sequences of VvmiR166a-h, and predicted their target HD-Zip III TFs (VVREV, VVHB15and VVHOX32) in grape cv. 'Rosario Bianco'. The complementary degree and cleavage role of diverse VvmiR166s-medicated VVREV, VVATHB15 and $V V H O X 32$ target genes, were further verified via an integrated approach of RNA ligase-mediated 5'-rapid amplification of cDNA ends (RLM-RACE) and poly(A) polymerase-mediated 3'-rapid amplification of cDNA ends(PPM-RACE). The promoter analysis of VvmiR166s and target genes( $V V R E V, V V H B 15$ and $V V H O X 32)$ reflected their potential roles in GA-medicated grape parthenocarpy. Subsequently, the spatio-temporal expression of VVmiR166s, and $V V R E V, V V A T H B 15$ and $V V H O X 32$ target genes was carried out in flesh, peel and seed of the grape berries at different flowering stages. Our investigation of GA-mediated VvmiR166 regulation of berry development will provide important information for seedless grape production, as well as other fruit trees, and also contribute to the molecular breeding of seedless grapes.

\section{Materials And Methods \\ Plant materials and hormone treatments}

Based on our preliminary study and the necessity of collected experimental berry samples, 5-year-old trees of grapevine cv.' Rosario Bianco' (Vitis vinifera L.), grown under common field conditions at the Jiangsu Vocational College of Agriculture and Forestry, grape farm, Jurong, China, were used as the plant material in this study. According to the production experience and variety characteristics, total 3 grape plants were randomly selected as experimental materials (one plant for each experiment, and three repeats). Three clusters of inflorescence of each plant were treated with $50 \mathrm{mg} / \mathrm{L} \mathrm{GA}$ for 30s, and other three clusters of the corresponding plants were done with water for 30 s as control at 10 days before flowering.Samples were collected at the 32, 46, 60 and 86days after flowering, part of the samples were used for measurement of physiological index, and the other part of samples were immediately frozen in liquid nitrogen and stored at $-80^{\circ} \mathrm{C}$ after separation for further investigation.

\section{Prediction the targets of VvmiR166s and their functions by bio-informatics}

The precise mature sequences of VvmiR166s were aligned by Multiple Align (BioXM software), revealing two unique mature sequences (VvmiR166a/b and VvmiR160c/d/e/f/g/h). Based on the complementary traits of miRNAs and their target genes sequences, together with the bio-informatics methods, we employ the psRNA Target Software online (http://plantgrn.noble.org/psRNATarget/) to predicate the target genes and interaction modes of VvmiR166a/b and VvmiR166 c/d/e/f/g/h, respectively. Furthermore, the function of target genes were annotated by the Grapevine Genome CRIBI Biotech website (http://genomes.cribi.unipd.it/).

\section{Motif analysis of the promoters of VvMIR166 s and VvHOX32, VvREV and VvHB15}

Based on the grape genome database, we obtain the promoters (approximately 1500 bp upstream of genes) of $V V M I R 166 s$ and $V v H O X 32, V_{V} R E V$, and $V V H B 15$, and employed the Plantcare software to predict the motif elements of these promoters. We further analyze the potential functions of these motif elements and compare the conservation of motif elements between those from the promoters of $V_{V} M I R 166 s$ and $V v H O X 32, V_{V} R E V$, and $V v H B 15$.

\section{Construction Of Phylogenetic Tree}

The software MEGA version 7.0 and Clustal X2 were employed to conduct the phylogenetic analysis. Homologous of $V v H O X 32, V_{V} R E V / V V R E V$-like and $V V H B 15$ were identified by a blast search of the NCBI databases (https://blast.ncbi.nlm.nih.gov/Blast.cgi) using nucleotide and amino acid sequences of $V v H O X 32, V_{V} R E V / V V R E V$-like and $V v H B 15$. Multiple alignments performed using ClustalX2, the unrooted phylogenetic trees were constructed using Neighbor-Joining (NJ)method, and the bootstrap test was carried out with 1000 iterations.

\section{Rna Extraction And Cdna Synthesis}

Total RNA was isolated from $200 \mathrm{mg}$ of the grapevine tissues mentioned above using the modified CTAB method. Low-molecular-weight RNA (LMW RNA) and high-molecular-weight RNA (HMW RNA) were separated with 4M LiCl. The concentration of RNA was measured by a UV-1800 spectrophotometer and visually ascertained in a $1.0 \%$ agarose gel. LMW RNA was polyadenylated at $37^{\circ} \mathrm{C}$ for 60 min in a $50-\mu \mathrm{L}$ reaction mixture with $1.5 \mu \mathrm{g}$ of total RNA, $1 \mathrm{mM}$ ATP, $2.5 \mathrm{mM} \mathrm{MnCl} 2$, and $4 \mathrm{U}$ poly(A) polymerase (Ambion, Austin, TX). The Poly (A)-tailed LMW RNA was recovered by phenol/chloroform extraction and ethanol precipitation. The Poly (A)-tailed LMW RNA were further ligatedto5'adapter (5'- 
CGACTGGAGCACGAGGACACTGACATGGACTGAAGGAGTAGAAA-3') using T4 RNA ligase (Invitrogen, Carlsbad, CA). The ligation products were recovered by phenol/chloroform extraction followed by ethanol precipitation. Reverse transcription was performed using $1.5 \mu \mathrm{g}$ of small RNA and $1 \mu \mathrm{g}$ of (dT)30 RT primer [ATTCAGAGGCCGAGGCGG CCGACATG-d(T)30 (A, G or C) (A, G, C, or T)] with $200 \mathrm{U}$ of SuperScript III reverse transcriptase (Invitrogen, Carlsbad, CA). Poly (A)-tailed small RNA or mRNA (10 $\mu \mathrm{L}$ total volumes) was incubated with $1 \mu \mathrm{L}$ of (dT)30 RT primer and $1 \mu \mathrm{L}$ dNTP mix (10 $\mathrm{mM}$ each) at $65^{\circ} \mathrm{C}$ for $5 \mathrm{~min}$. The reactions were chilled in ice for at least $2 \mathrm{~min}$, then the remaining reagents $(5 \times$ buffers, dithiothreitol (DTT), RNase out, SuperScript III)were added as specified in the SuperScript III manual, and the reaction was left to proceed for 60 min at $50^{\circ} \mathrm{C}$. Finally, the reverse transcriptase was inactivated by incubation for $15 \mathrm{~min}$ at $70^{\circ} \mathrm{C}$, and cDNA for mRNA and poly(A)-tailed small RNA was stored at $-20^{\circ} \mathrm{C}$ before use.

\section{Expression Analysis Of Vvmir166s By Qrt-pcr}

The cDNA for poly(A)-tailed small RNA mentioned above was used as template for quantitative real-time polymerase chain reaction (qRT-PCR). To amplify the VvmiR166a/b and VvmiR166c/d/e/f/g/h from the reverse-transcribed cDNAs, qRT-PCR was performed using SYBR Premix Ex TapTM kit (Takara, Dalian, China), and was performed with the Light Cycler®480 II (Roche, Switzerland). PCR cycling conditions consisted of an initial denaturation step at $95^{\circ} \mathrm{C}$ for $30 \mathrm{~s}$, followed by 40 cycles at $60^{\circ} \mathrm{C}$ for $20 \mathrm{~s}$ and $95^{\circ} \mathrm{C}$ for $5 \mathrm{~s}$. The $5.8 \mathrm{~s}$ rRNA was used as a reference gene (Table 4). Relative expression level was calculated with the formula $2^{-\triangle \Delta C T}=$ normalized expression ratio. Each PCR assay was carried out by three biological replicates, and each replicate corresponded to three technological repeats of separate experiments.

\section{Expression analysis of VvHOX32, VvREV and VvHB15}

The expression of $V V H O X 32, V V R E V$, and $V V H B 15$ was assayed by qRT-PCR as previously described. The reverse transcription product was amplified using gene-specific primers that overlapped the known or predicted cleavage site. Reactions were performed in triplicate on the Light Cycler ${ }^{8} 80$ II (Roche, Switzerland). The UBI gene was used as a reference gene in the qRT-PCR detection of mRNAs (Table 4). Relative expression level was calculated with the formula $2^{-\triangle \Delta C T}=$ normalized expression ratio. Each PCR assay was carried out in three biological replicates, and each replicate corresponded to three technological repeats of separate experiments.

\section{Mapping Mrna Cleavage Sites With RIm-race And Ppm-race}

To map miRNA-mediated cleavage products, we developed and performed an integrated method comprising the following steps: HMW RNAs were polyadenylated at $37^{\circ} \mathrm{C}$ for $60 \mathrm{~min}$ in a $50 \mu \mathrm{l}$ reaction mixture with $5 \mu \mathrm{g}$ of HMW RNAs, $1 \mathrm{mM} \mathrm{ATP}, 2.5 \mathrm{mM} \mathrm{MnCl} 2,5 \mathrm{mM} 5 \times$ buffer, and $8 \mathrm{U}$ poly(A) polymerase (Ambion, Austin, TX), and HMW RNAs were ligated to a 5】 adapter (5囚-CGACUGGAGCACGAGGACACUGACAUGGACUGAAGGAGUAGAAA-3囚) using T4 RNA ligase (Invitrogen, Carlsbad, CA), respectively. Then Poly (A)-tailed HMW RNA and adapter-ligated HMW RNA were recovered by phenol/chloroform extraction followed by ethanol precipitation. Reverse transcription was performed using $5 \mu \mathrm{g}$ of Poly (A)-tailed HMW RNA and adapter-ligated HMW RNA, respectively, $1 \mu \mathrm{g}$ of (dT)30 RT primer (ATTCTAGAGGCCGAGGCGGCCGACATG-d(T)30 (A, G, or C) (A, G, C, or T)) with $200 \mathrm{U}$ of SuperScript III reverse transcriptase (Invitrogen, Carlsbad, CA). Poly (A)-tailed HMW RNA and adapter-ligated HMW RNA (10 $\mu$ l total volume) were respectively incubated with $1 \mu \mathrm{l}$ of (dT) $30 \mathrm{RT}$ primer and $1 \mu \mathrm{dNNTP}$ mix ( $10 \mathrm{mM}$ each) at $65^{\circ} \mathrm{C}$ for 5 min to remove any RNA secondary structures. The reactions were chilled on ice for at least $2 \mathrm{~min}$, then the remaining reagents [5× buffers, DTT, RNase out, SuperScript III] were added as specified in the SuperScript III manual, and the reaction proceeded for $60 \mathrm{~min}$ at $50^{\circ} \mathrm{C}$. Finally, the reverse transcriptase was inactivated for $15 \mathrm{~min}$ incubation at $70^{\circ} \mathrm{C}$. After the preparation of miRNA-cleaved target mRNA libraries from various organs and tissues, we pooled similar quantities of these library samples for further PCR and qRT-PCR amplification reactions. The PCR amplifications were performed using the Gene Racer $5 \rrbracket$ primer and the gene-specific primers. The amplification products were gel purified, cloned, and sequenced, and at least eight independent clones were sequenced.

\section{Sub-cellular Localization}

The gene sequences of $V V H B 15, V_{V} R E V$ and $V V H O X 32$ were amplified by PCR using the primer in table S1. $V_{V} H B 15, V_{V} R E V$ and $V_{V} H O X 32$ were integrated into the pCAMBIA1302 binary vector by double digestion (35S-VVATHB15:GFP, 35S-VVREV:GFP and 35S-VVHOX32:GFP). 35S-GFP was used as a blank control. The binary vector was transiently transformed into tobacco by Agrobacterium-mediated method, and after 3 days of dark culture, sub-cellular localization was observed using laser con-focal.

\section{Construction of the expression vector and Agrobacterium-mediated tobacco transient transformation}

The gene sequences of $V V M I R 166 s$ and $V V H B 15$ were isolated. The primers used were described in tables S2. To develop pCAMBIA1302- $V V M I R 166 s$ and pCAMBIA1302-VVATHB15 constructs, 500-600bp VVMIR166s and 2523bp VVHB15 were independently cloned and integrated into the pCAMBIA1302 binary vectors, respectively. A single colony of Agrobacterium tumefaciens EHA105 (containing the recombinant plasmid) was cultured in LB liquid medium provided with rifampin $\left(50 \mu \mathrm{g} \mathrm{mL}^{-1}\right)$ and kanamycin $\left(50 \mu \mathrm{g} \mathrm{mL}^{-1}\right)$ and culturing to OD600 $=0.5$. The bacteria were then pelleted and re-suspended in suspension buffer $(10 \mathrm{mM} \mathrm{MES}, 10 \mathrm{mM} \mathrm{MgCl} 2, \mathrm{pH} 5.6)$. The bacterial suspension was then adjusted to 0 D600 $=0.8$, and 100 $\mu \mathrm{mol}$ of $\mathrm{L}^{-1}$ acetosyringone was added before the suspension was used for permeation, and allowed to stand at room temperature for 4 hours. The bacterial suspension was immersed by injection into the leaves of 6 week old tobacco plants. The infiltrated seedlings were then moved back to the environmental chamber and placed in the dark for 3 days. 


\section{Results}

\section{Morphological and physiological changes of grape berries in response to GA application}

To investigate the effects of GA on the grape berry and seed development, the morphological and physiological variation of grape cv. 'Rosario Bianco' was observed and examined. GA treatment conspicuously induced seedless berries with nearly $99 \%$ seedless ratio, while GA-untreated control plants had no seedless berries (Table 1 and Fig. 1). In addition, GA treatment distinctly affected the fruit shape index of grape berries, which increased from 0.26 to 0.43 along with the development of grape berries. GA-treated grape berries exhibited the longer fruit brush in contrast with control ones, and the smaller diameter of fruit brush relative to control plants (Table 1 and Fig.1). These morphological changes reflected that some physiological and molecular responsive to GA might be involved in the modulation of grape berry development.

\section{Identification of VvmiR166s in grape cv. 'Rosario Bianco' by miR-RACE and their homologous sequences comparison with those in miRBase}

miR-RACE technology was employed to verify VvmiR166a/b/c/d/e/f/g/h precise sequences in grape cv. 'Rosario Bianco'. Our results demonstrated that the cloned sequences of all VvmiR166 family members were identical in length (21nt), and VvmiR166a/b were different from the validated miRNAs in miRbase 21.0 (Fig. 2). The sequence analysis showed that VvmiR166a exhibited two more bases "TC" at the 5'-end, while VvmiR166a had two more bases "TC" at the 3'-end (Fig. 2A). However, the identified sequences of the remaining members VvmiR166c/d/e/f/g/h were similar with those of homologous sequences in miRBase 21.0 (Fig. 2A). These results indicated that there is diversification as well as conservation in the sequences of miR166 family members. The determination of VvmiR166s precise sequences could provide significant base for prediction of their target genes, which contributed in recognizing the VvmiR166s roles in grapes. In contrast with the similarity of the mature sequences between VvmiR166 family members, their precursor sequences were less similar based on the alignment analysis. These eight VvmiRNA precursor sequences (VVMIRNAs) were divided into two groups, including $V_{V M I R} 166 a / b$ and $V v M I R 166 \mathrm{c} / \mathrm{d} / \mathrm{e} / \mathrm{f} / \mathrm{g} / \mathrm{h}$ with $50 \%$ and $36 \%$ similarity, respectively (Fig. $2 \mathrm{~B}$, red box regions); while all VvmiR166s possessed about $90 \%$ identity at their mature sequences, confirming VvmiR166s had the higher conservation than corresponding $V v M I R 166 s$.

\section{Analysis of sequences complementary degree and potential cleavage roles between VvmiR166s and their predicated target genes}

The precise sequences of VvmiR166s were used as queries to search their target genes from grape mRNA database (http://genomes.cribi.unipd.it/DATA/V2/V2/). In total, four genes VvHOX32, VvREV, VvReloluta-Like, and VvHB15 were predicted as target genes for each member of the VvmiR166 family, and all target regions of VvmiR166 family members were located in the coding sequences (CDS) of their target genes (Table 2). Further functional annotation of target genes showed that $V v H O X 32, V_{V} R E V, V_{V} R e l o l u t a-L i k e$, and $V_{v} H B 15$ belong to the HD-ZIP III class of HD-Zip TF family (Table3). Analyzing the complementary degree of VvmiR166a/b/c/d/e/f/g/h and $V v H B 15, V v R E V, V v R e / o l u t a-L i k e$ and $V v H O X 32$ target genes, the result depicted that VvmiR166a/b displayed one mismatch base with VvHB15, VvREV, VvReloluta-Like and $V v H O X 32$, while $V v m i R 166 \mathrm{c} / \mathrm{d} / \mathrm{e} / \mathrm{f} / \mathrm{g} / \mathrm{h}$ and target genes had three mismatch bases (Fig. 3), indicating that VvmiR166a/b might have the stronger cleavage roles on these four target genes than VvmiR166c-h. Interestingly, we also revealed that all these mismatched positions between VvmiR166s and their targets almost occurred at both ends of VvmiR166s sequences. The result of high conservation at the sites with mismatch bases between miR160s and target genes was in line with other reports(Li and Mao 2007; Cuperus et al. 2011; Floyd and Bowman 2004), suggesting that this consistency might be one of the reasons for the conservation of miRNA-targeted modes within different plant species.

\section{Analysis on motifs of promoters of VVMIR166s and their potential target genes}

The promoter plays the key role in the initiation of transcription process, and its sequence contained some essential functional components (motifs), indicating the potential functions of genes. Here, we analyzed the motif elements of VvMIR166s and VvHB15, VvRevoluta, VvRevoluta-like, and $V v H O X 32$. To conveniently depict the traits of these gene promoters, all motifs were classified into five different types, including light, hormone, tissuespecific, circadian and stress responsive elements. As shown in Fig. 4A, VVMIR166s almost has five kinds of cis-elements expect for $V V M I R 166 e / f$ without circadian responsive elements, and among these five cis-elements, the number of light-related elements is the largest, accounting for more than $1 / 3$ of all elements contained, which might derive from the fact that the light is necessary for photosynthesis in all green plants. Contrarily, all four target genes of VvmiR166s have the similar motif elements, of which $V v R E V$-like and $V v H O X 32$ contained 5 types of cis-elements above, while $V V R E V$ and $V V H B 15$ had 4 out of 5 kinds of cis-elements without circadian-related genes, implying their potential functions in these aspects mentioned above.

Our earlier work revealed that VvmiR166 family could conspicuously respond to GA(Han et al. 2014), and thus, we further analyzed the motifs related to hormones for the promoters of VVMIR166s and target genes. As shown in Fig. 4B, the promoters of $V_{V M I R} 166 \mathrm{~s}$ and target genes consist of motifs responsive to hormones like GA, abscisic acid (ABA), Ethylene(ET), methyl jasmonate (MeJA), salicylic acid(SA) and auxin. Interestingly, the promoters of $V v M I R 166 \mathrm{a} / \mathrm{b} / \mathrm{c} / \mathrm{d} / \mathrm{e} / \mathrm{g} / \mathrm{h}$ and $V_{v} H B 15$ contained cis-elements responsive to GA. SA responsive elements were predicted in the promoters of $V V M I R 166 a / b / c / d / e / / h$ and $V v H B 15$. Motifs responsive to ABA were identified in the promoter regions of $V v M I R 166 a / c$ and $V v H O X 32$, while those responsive to MeJA were observed in the promoters of $V V M I R 166 \mathrm{~b} / \mathrm{e}$ and $V V H B 15$.None of the promoters of $V V M I R 166 \mathrm{family}$ memebers possessed motifs responsive to auxin, while target genes $V v$ Revoluta, $V v R$ Revoluta-like, and $V v H O X 32$ had auxin-related motifs. These results suggested that VvmiR166 family and target geneVvHB15 might be mainly involved in the modulation of GA and SA, while GA and SA played the significant roles during grape berry and seed development, and thus we deduced VvmiR166 family and $V v H B 15$ might involve in the regulation of grape berry and seed development by responding to GA and SA signal. On the other hand, we also observed that the diverse members of the VvmiR166 family might possess 
the redundancy and complementary of functions in responding hormones. The promoters of VVMIR166s and their target genes exhibited the diverse traits in the types and numbers of motifs.

\section{Clone, sequence analysis and sub-cellular localization of target genes of VvmiR166s}

Based on the predicted target mRNA sequences of VvmiR166s, we cloned and identified the sequences of $V_{V} H O X 32, V_{V} R E V$ and $V V H B 15$ in the berries of grape cv. 'Rosario Bianco'. $V V R E V$ had an open reading frame (ORF) of 2532 bp encoding a protein of 844 amino acid residues, and the ORF of $V v H O X 32$ was 2535 bp encoding a protein of 845 amino acid residues, while $V v H B 15$ possessed 2520 bp ORF encoding a protein of 840 amino acid residues (Fig. 5A). These three proteins all comprised of four conserved domains, including homeo domain(HD), leucine-zipper domain (Zip), steroidogenic acute regulatory protein-related lipid transfer domain (START), and MEKHLA domain (Fig. 5A). We further compared the amino acid sequences of our VvHOX32, VvREV, and VvHB15 proteins encoding genes with their homologous proteins derived from Arabidopsis thaliana, Nicotiana tabacum, Fragaria vesca ssp. Vesca and Prunuspersica (Fig. 5B), and the phylogenetic tree was constructed using the MEGA software V.7 with the neighbor-joining algorithm (Fig. 5C). The Gene Structure Display Server (GSDS, http://gsds.cbi.pku.edu.cn/index.php) was also employed to analyze the intron, exon and up/downstream UTRs. The targrt proteins VvREV, VvHOX32, and VvHB15 belong to HD-Zip III family, and their phylogenetic analysis divided them into the three subgroups. VvHB15 had the closest relationship to the PtHB15, while VvHOX32 was highly similar to NtHOX32, but VvREV located at one small branch far from other homologous genes in other plant species (Fig. 5C), indicating that the various members of same gene family might be involve in diverse evolution process. Further analysis of sequence structure revealed that the extra and intron number of these three target genes are similar, while they are different in the distribution in chromosomes. $V V R E V, V v H O X 32$ and $V v H B 15$ exhibited longer introns in length and looser structures of their sequence in contrast with homologous genes of other five plant species, implying that the divergence during their evolution process (Fig. 5D). Sub-cellular localization analysis of $V V R E V, V v H O X 32$ and $V v H B 15$ with the 35S-VVREV/VVHOX32/VVHB15:GFP and 35S-GFP being transiently expressed in tobacco leaf epidermal cells showed that the fluorescence in the tobacco cell trans-formed with $35 S-V V R E V: G F P$ was localized both in the cytoplasm and in the nucleus; 35S-VVHOX32:GFP was detected exclusively in cytoplasm, while 35S-VVHB15:GFP was just localized in the nucleus (Fig. 6). The divergence in their Sub-cellular localization implied the difference of their potential functions.

\section{Verification of VvmiR166s-guided cleavage roles on their target mRNAs in grapes}

The detection of the cleaved products of target genes for miRNAs is necessary to study the miRNAs regulation mechanism on their target genes. miRNAs negatively regulate the target genes through miRNA-directed cleavage within the region of complementary or interfering with translation. Most of the plant miRNAs have been shown to guide cleavage of their target genes. To examine whether the three predicted targets of $V v m i R 166 s$ can also be cleaved, our modified RLM-RACE technology and developed PPM-RACE procedure were used to map the cleavage sites in predicted target genes from grapevines. All the anticipated RLM-RACE products (3'-end cleavage mRNAs) showing distinct bands on agarose gel were isolated and sequenced, and the $3^{\prime}$-end cleavage mRNA sequences could be mapped into $V v H O X 32, V_{V} R E V$, and $V v H B 15$.Thus these three genes could be validated as the target genes ofVvmiR166s, and their cleavage sites mainly happened at the $9^{\text {th }}$ site from $5^{\text {'-end }}$ of VvmiR166s at the binding sites, which was similar to previous reports (Fig. 7). Subsequently, PPM-RACE was further employed to verify these cleavage roles and cleavage sites. The results showed that PPM-RACE products (5'-end cleavage mRNAs) were also mapped into $V v H O X 32, V V R E V$, and $V v H B 15$, and their cleavage sites also mainly occurred at the $9^{\text {th }}$ position. The consistency of cleavage sites of RLM-RACE and PPM-RACE further confirmed these genes to be the true targets ofVvmiR166s, and their negatively regulatory roles of VvmiR166s on these three target genes.

\section{Spatio-temporal Expression patterns of VvmiR166s and their target mRNAs $V v H O X 32, V v R E V$, and $V v H B 15$ during diverse grape berry tissues development}

Expression profiles of miRNAs in various tissues not only confirm the existence of the miRNAs in the organisms but also provide clues about their physiological functions. To recognize the potential functions of VvmiR166s, qRT-PCR analysis was employed to detect the expression levels of VVMIR166s genes (VvmiR166s precursors) and their targets (the primer in Table.4) in the various tissues of grape berries for a more efficient characterization of VvmiR166s and their target genes. The results of qRT-PCR clearly indicated that VvmiR166s were expressed ubiquitously in three types of grape berry tissues (peel, flesh, and seed), showing some tissue and/or stage-specific patterns. As shown in Fig. 8, almost all VvmiR166s possessed a typical character of the lowest expression in grapevine stone hardening stage (46 DAF), while exhibited the ubiquitous expression level in other stages. For instance, VvmiR166d had the high expressions in the seeds of the remaining stages, followed by VvmiR166a, VvmiR166g and VvmiR166h, indicating these members might involve in the modulation of the development of the special stages. The expression of VvmiR166b/c/e exhibited the "V"mode from the 32DAF to 80DAF during the development of grape berries, the lowest expression level was detected at 46DAF in the corresponding tissues, suggesting that regulatory roles of VvmiR166b/c/e might possess the dynamic variation during grape berry development. Unlike the above two expression modes, VvmiR166f had the high expression levels in the seed and flesh at the 32DAF, while exhibited the low expression levels at the other stages, indicating VvmiR166f might mainly involve in the modulation of the special stage of grape berry development.

To further gain insight into the roles of VvmiR166s in grape berries, the expression levels of their target genes were analyzed. As shown in Fig. 9, three target genes of $V_{V R E V}, V_{V} H B 15$, and $V v H O X 32$ exhibited the lowest expression levels in flesh tissues at various development stages of grape berries. $V_{V} R E V$ and $V V H B 15$ possessed the highest expression levels in the seed tissues at various development stages, while $V V H O X 32 \mathrm{had}$ the higher expression levels in the peel tissues at different stages than the flesh and peel tissues. As described above, these three target genes belong to HD-Zip III family involved in modulation of vascular and xylem formation, we deduced that they might involve in regulating vascular and xylem formation of seed stones and peels of grape, and highly expressed in the whole seeds and peels. 
Compared to the correlation of $V v m i R 166 s$ and their targets at the expression levels, we revealed that VvmiR166f and $V v H O X 32$ had the reverse expression modes during grape berry development, implying VvmiR166f might negatively regulate the expression of the $V v H O X 32$. Besides this, the remaining VvmiR166 family members showed the negative correlation with these three target genes at their expression levels in the certain tissues at some special stages of grape berry development, like in seed and peel tissues at the 46DAF and 60DAF (Fig. 8 and Fig. 9). Considering the redundancy and complementary traits of function of miRNA, the total expression levels of all members of VvmiR166 family were detected to compare to each target gene,the results showed that except for $V v H O X 32$ in peel tissues, VvmiR166shad negative correlations with three target genes at their expression levels in special periods of various tissues (See* marked in Fig. 10). For instance, there were negative relationships in some extent betweenVvmiR166sand $V v H B 15$ and $V V R E V$ in seed tissues during the later period of grape development (Fig. 10 * marked), while the expression modes of VvmiR166s and $V v H O X 32$ were similar at the former stages (Fig. $10 *$ marked). In addition, VvmiR166s and target genes also showed opposite expression trend in peel and flesh tissues at multiple development stages (see Fig. 10 * marked). These results further presented some evidence to support the negative regulatory roles of VvmiR166s on three target genes in some special stages of grapes.

\section{Modes of VvmiR166s and their target mRNAs responding to GA during the development of grape berries}

GA plays the significant roles in grape berry development, and our previous study showed VvmiR166s could respond to exogenous GA(Han et al. 2014). In the present study, the modes of VvmiR166s and their target mRNAs responsive to GA in various tissues of berries during grape berry development were further investigated. The results showed that in seed tissues, the expression of VvmiR166a/b/f/g were down-regulated by GA in almost all stages, depressed at the lowest levels at 46DAF. The remaining members exhibited the diverse variation trends during diverse stages of seeds development, implying that GA might modulate seeds development mainly by inhibiting the expressions of VvmiR166a/b/f/g. In contrast, VvmiR166b/c/h was down-regulated by GA only at the former berry development stages in peel tissues, like 32DAF and 46DAF. The remaining members were up-regulated or exhibited on change in expression level under GA treatment in all stages. GA treatment reduced $V v m i R 166 c / d / f / h$ expression at 32DAF in flesh tissues, while there was no significant effect of GA treatment on the remaining members in all stages (Fig. 11). All these results confirmed that VvmiR166 family possessed the tissue and stage-specific characters as well as diversification in responding to $\mathrm{GA}$.

In contrast with VvmiR166s, their target genes exhibited the differential expression modes responsive to GA in grape berries (Fig. 12). The two interesting cases were observed among them, in one case, GA treatment induced $V v H B 15$ expression at 46 DAF in seeds and peels, especially reverse to those of several members of VvmiR166 family in seeds, indicating GA manipulated seeds development possibly by repressing the expressions of VvmiR166to promote $V v H B 15$ expression. Another case is that all these target genes were obviously up-regulated by GA at all stages in fresh tissues. These findings demonstrated that these target genes possessed diverse modes responsive to GA in grape fresh tissues with those in corresponding seeds and peels, and meanwhile suggesting VvmiR166 family might negatively modulate $V v H B 15$ expression in the special tissues of the certain stage grape berries by responding to GA. Our findings confirmed that VvmiR166 family possessed the characterization of stage/tissue-specific involved in the grape berry development in response to GA by mediating their target genes.

\section{Effect of GA on the regulatory modes of VvmiR166s:VvHB15 during GA-induced grape seedless berry development}

From the analysis above, it is revealed that VvmiR166: VvHB15 module might be one of key regulators amongst VvmiR166 family during GA-induced grape seedless berry development. To further recognize the effect of GA on regulatory modes of Vvmi166: $V v H B 15$ pair, we analyzed the variation of GA manipulating on the expression correlation of VvmiR166s and VvHB15. Considering of the stage specificity of VvmiRNAs and target genes at their expression levels, the two special important developmental periods of 32DAF-46DAF (from young to stone hardening stage, called as stage I) and 60DAF-86DAF (from color turning to mature stage, called as stage II) were selected for investigation of the expression correlations between $V v m i R 166 s$ and $V v H B 15$. As Fig. 13 showed, VvmiR166s and $V v H B 15$ had apparent positive correlations $(r=1)$ at stage I of seed, peel and fresh tissues in control plants, while part members of VvmiR166 family and $V v H B 15$ exhibitednegativecorrelation( $r=-1)$ at stage II of corresponding tissues (See the blue $*$ ). The result indicated that VvmiR166s might play roles at stage II of seed, peel and fresh tissues through negatively modulating the expression of $V v H B 15$. Interestingly, VvmiR166 family members and $V v H B 15$ expression possessed significant negative correlation ( $r=-1$ ) under GA treatments in comparison with untreated control plants(See red $※$ ), suggesting VvmiR166 family could be modulated by GA to negatively mediate the expression of $V V H B 15$ mainly at stage I of grape seed and peel tissues. These results confirmed that GA could efficiently change the regulatory modes of VvmiR166s on $V v H B 15$, where their negatively modulated roles occurred at from stage II (CK) to stage I(GA treatment).

On the other hand, we also revealed that during GA-induced grape seedless berry development, GA could up-regulated the expressionsofVvmiR166 members at seeds, leading to inhibition of $V v H B 15$ expression, while previous studies showed ArabidopsisAtHB15-OE could inhibit lignin synthesis(Boualem et al. 2008), thus we deduced VvmiR166s: VVAtHB15 pairs might be one of reasons that GA repressed grape seed stone development to induce grape seedless berries. This could provide the significant clue for further elucidating the molecular mechanism of GA-induced grape seedless berry.

\section{Roles of VvmiR166s-mediated $V v H B 15$ in modulation of lignin synthesis process}

To recognize the potential function of VvmiR166s and $V v H B 15$, we constructed their corresponding transient over-expression pCAMBIA1302 vector ( VvMIR166s/VvHB15-OE) (Fig.14A), and the empty pCAMBIA1302 vector was used as the control. These vectors were transiently transformed into tobacco plants using an Agrobacterium-mediated method. After dark culture of 3 days, DNA was extracted, and specific primers were used for PCR 
detection. The precise sequences after gel electrophoresis and sequencing were identical to purpose ones (Fig.14B), confirming the transient overexpression of VVMIR166s/VVATHB15 in tobacco plants (Fig.14C).

In view of the potential functions of HD-ZIP III transcription factors in the modulation of lignin synthesis of mode plants(Boualem et al. 2008), we further investigated the effect of over-expression of $V v M I R 166 s / V v H B 15$ on the expression levels of genes (NtPAL, NtCCR, NtCAD, NtLaccase, Nt4CL, $N t 4 C L, N t C O M T$ ) involved in the lignin synthesis pathway. Results showed that among VVMIR166 family, VVMIR166h might be one of main regulators in this process, over-expression of VVMIR166hin tobacco plantsevidently up-regulated the expression of NtPAL4, NtCCR1, NtCOMT1 and NtCCOAOMT6, followed by $V V M I R 166 b$ (markedly up-regulated those of $V V L a c c a s s e 3$ and $V V L a c c a s e 17$ ) and $V V M I R 166 a$ (observably up-regulated that of $V V C A D 1$ ), while over-expression of $V v H B 15$ obviously down-regulated the expression of NtPAL4, NtCCR1 and NtCAD1(Fig. 14D), indicating VvMIR166s possessed the opposite roles to $V V H B 15$ in modulation of lignin synthesis. Furthermore, the transient over-expression of VvmiR166s in Tobaccos obviously decreased the expressions of NtHB15 and NtHB15-likegenes, which were high conserved with VvHB15, especially complete consistent VvmiR166stargeted regions in these three orthologous genes. Together with the modes of VvmiR166s and their targets responsive to GA, it was revealed that the potential roles of VvmiR166b/h during GA-induced grape seedless berry process might function to inhibit grape seed-stone development through the key signal pathway of "GA-VvmiR166h/b-VvHB15 tignin synthesis genes" (Fig.15). All these results confirmed that VvmiR166b/h might modulate the synthesis of lignin mainly by negatively regulating $V v H B 15$ in grapes (Fig.14E), which was in line with previous studies by Boualem (Boualem et al. 2008).

\section{Discussion}

miRNA-based molecular studies have set provide bases for analyzing the evolution and functions of miRNAs from a phylogenetic perspective. Evolutionary conservation is a characteristic feature that consents identification of miRNAs homologs in previously reported ones from other species. miRNAs is characterized as the temporal and spatial expression patterns and play a fine-tuning role primarily at the post-transcriptional level by regulating the expression of the protein-coding genes. Although lots of GA-responsive miRNAs in grapevine have been studied in our previous work(Han et al. 2014), their functions in grapes are still unclear. In this study, we reported the interaction mechanism of VvmiR166s and their target mRNAs in response to GA to modulate the development of the seedless grape berries.

Here, four genes $V v H B 15, V v R E V, V V R E V$-like and $V v H O X 32$, encoding putative ClassIII HD-Zip proteins, were predicted as the target genes of VvmiR166s in grapes. The bioinformatic analysis demonstrated that all targeted regions of VvmiR166s on their target genes located in the CDS regions of the corresponding targets, and VvmiR166s interacted with their target genes with the cleavage modes, which was further verified by our RLM-RACE and PPM-RACE experiments (Fig. 7). These results resembled the previous reports where plant miRNAs modulated their target genes mainly by the cleavage modes(Depuydt and Hardtke 2011), demonstrating that VvmiR166 family directly targeted on REV,ATHB15and HOX32 transcription factors in the HD-ZIP family,and involved in modulating plant growth and development processes, including the shoot apical meristem, floral meristem embryo, vascular and xylem formation(Leung and Sharp 2010; Bushati and Cohen 2007; Jonesrhoades and Bartal 2002; Palatnik et al. 2003).

The promoter motifs of a gene could provide significant information for the prediction of potential functions. We observed that the promoters of all VVMIR166s and their target genes had the largest number of motifs response to light (37-71\%; $43-62 \%)$, as well as to stress (9-30\%; $17 \%-26 \%)$, hormone $(4-24 \% ; 5-19 \%)$ and specific tissue (4-22\%; 6-20\%), indicating that they might possess some potential functions in these aspects. It is possible that light, as an essential element for plant growth, plays an irreplaceable role in the growth and development of grape berries. Motifs responded to hormones are also important cis-acting factors, the promoters of $V v H B 15$ and $V v M I R 166 a / b / c / d / e / g / h$ have GA cis-acting factors (Fig. 4). Analyzing the relative expression of the target genes and VvmiR166s (Fig. 8 and Fig. 9), our study revealed that all VvmiR166s possessed one typical character with the lowest expression at grapevine stone hardening stage and higher expressional value of the target gene in lignified tissues (seed and peel). As shown in Fig. 10, it was revealed that the expression of VvmiR166 family members exhibited the negative correlation with their target genes in different tissues treated by exogenous GA (Fig. 11 and Fig. 12). GA treatment down-regulated the expressions of some VvmiR166 memebers especially in seed tissues, and increased the expression of target genes. We can infer exogenous GA treatment mediates VvmiR166 family and acts on the target genes to guide grape seedlessness.

The functions reported in previous studies could provide some evidence supporting potential functions of these genes as predicted by motif analysis in this work. Based on the phylogenetic tree of homologous genes across various plant species, we could presume the characterization of conservation and diversification of these genes, and construct the potential functions of some homologous genes. In this study, three target genes of Class III HD-Zip can be further classified into the diverse sub-groups, more importantly, their evolution processes were innumerable. For instance, $V V R E V$ and other homologous REV genes in other plant species categorized into one group, while VVHB15 and homologous AtHB15, AtHB8 into other one group, similarly, $V V H O X 32$ and orthologous $H O X 32, A t H B 14, P H B$ into another group. These findings could provide significant information for further understanding the functions of these three target genes based on their conservation with the homologous genes of model plants. Previous studies have shown that $R E V$ is involved in the regulation of embryo development, vascular tissue formation, polar auxin transportion, plant morphology and development. $V V R E V$ was genetically more closed with homologous REV genes in other plant species, indicating $V V R E V$ might possess similar functions. On the other hand, we also revealed that $V V R E V$ had the highest expression levels in grape seeds, signifying the potential functions in modulation of grape seed development (it will be researched in our next work).

As described above, over-expression of AtHB15 inhibited the accumulation of lignins (Taku and Fukuda 2007), our work also unveiled that the transient over-expression of $V v H B 15$ in Tobaccos down-regulated the expressions of genes in lignin synthesis, while over-expression VvMIR166s promoted the 
expression of lignin synthesis genes. Moreover, GA repressed the accumulation of lignins in seeds of grape berries through up-regulating VvmiR166s to inhibit the expression of $V v H B 15$. These two results supported the view that $V v H B 15$ might be one important regulatory factor in modulation of lignin accumulation in grape seed stone tissues, which was identical with the previous reports(Taku and Fukuda 2007; Bang et al. 2018; Zhang et al. 2018). Repression of secondary cell walls formation in the pith is under the control of the class III HD-ZIP ATHB15 TF(Zhang et al. 2018), AtmiR166s-mediated cleavage of AtHB15 mRNA regulated vascular development in Arabidopsis inflorescence stems(Kim J. 2015), and an ectopic lignification phenotype was reported in the athb15 mutant(Cassanwang et al. 2013). Over-expression of miR166 could resulted in the down-regulation of target HD-ZIP IIIs transcript levels(Singh et al. 2014) and over-expression of VVMIR166s regulated the expressions of genes in lignin synthesis pathway by mediating target genes. Reversely, GA might inhibit the lignin synthesis to induce grape seedless berries by repressing VvmiR166sexpressions during grape berry development, especially at seed development. All these results supported the view that VvmiR166s mediated target genes involving in grape seed and stone development.

\section{Abbreviations}

$4 \mathrm{CL}$

4-coumarate CoA ligase

ABA

abscisic acid

AP2

apetala2

ATHB

arabidopsis thaliana homeobox

CAD

cinnamyl alcohol dehydrogenase

CCOAOMT

caffeoyl coenzyme A-O-methyltransferase

CCR

cinnamoyl CoA reductase

CDS

coding sequence

COMT

caffeic acid 0-methyltransferase

CTAB

cetyl-trimethyl-ammol/lonium bromide

DAF

days after flowering

GA

gibberellins

GFP

green fluorescent protein

HD

homeo domain

HD-Zip III

class III homeo domain-leucine zipper

HMW RNA

high-molecular-weight RNA

LMW RNA

low-molecular-weigut RNA

MeJA

methyl jasmonate

miRNAs

MicroRNAs

NCBI

national center for biotechnology information

nts

nucleotides

ORF

open reading frame

PAL 
phenylalanine ammonia-lyase

PCR

polymerase chain reaction

PHB

phabulosa

PHV

phavoluta

PPM-RACE

poly $(A)$ polymerase-mediated 3'-rapid amplification of cDNA ends

qRT-PCR

quantitative real-time-polymerase chain reaction

REV

revoluta

RLM-RACE

RNA ligase-mediated 5'-rapid amplification of cDNA ends

SA

salicylicacid

SPL

squamosa-promoter binding-like

START

steroidogenic acute regulatory protein-related lipid transfer domain

TF

transcriptional factors

UTR

untranslated region

Zip

leucine-zipper domain.

\section{Declarations}

\section{Acknowledgements}

The authors are grateful for the research laboratory facilities provided by the College of Horticulture, Nanjing Agricultural University, Nanjing, China. MA Professor would like to thank Tottori University, Japan for this collaborative research work.

\section{Funding}

This work was supported by the National Key Research and Development Program of China (2018YFD1000106), Jiangsu Province Natural Science Funds (BK20181318, BK20160587),National Natural Science Funds (31972373) and Central University basic scientific research business fee independent innovation major project (NATURAL SCIENCE) (KYTZ201602).

\section{Competing Interests}

The authors declare that they have no competing interests.

\section{Authors Contributions}

CW and JGF conceived and designed research. YHB, XXX, and XWZ conducted experiments. AM, ZJL and CZ contributed new reagents or analytical tools. WT, ZQX and TYD analyzed data. YHB and XXX wrote the manuscript. All authors read and approved the manuscript.

\section{Ethics approval and consent to participate}

The plants used in our study are not endangered species. Plant sample collection and transgenic plants were performed in accordance with the local legislation in China.

\section{Availability of data and materials}

All VvmiR166s and $V_{V} R E V, V_{V} A T H B 15$ and $V v H O X 32 \mathrm{cDNA}$ are available upon request. All primers and amino acid sequences used in this study are listed in Additional files 2: Tables S2 and Fig.5.

\section{References}


1. Abdelrahman M, Elsayed MA, Sato S, Hirakawa H, Ito S, Tanaka K, Mine Y, Sugiyama N, Suzuki Y, Yamauchi N, Shigyo M. RNA-sequencing-based transcriptome and biochemical analyses of steroidal saponin pathway in a complete set of Allium fistulosum-A. cepa monosomic addition lines. Plos One. 2018;12:e0181784.

2. Acheampong AK, Zheng CL, Halaly T, Giacomelli L, Takebayashi Y, Jikumaru Y, Kamiya Y, Lichter A, Or E. Abnormal endogenous repression of GA signaling in a seedless table grape cultivar with high berry growth response to GA application. Frontiers in Plant Science. 2017;8:850.

3. Bang SW, Lee D, Jung H, Chung PJ, Kim YS, Choi YD, Suh J, Kim J. Overexpression of OsTF1L, a rice HD-Zip transcription factor, promotes lignin biosynthesis and stomatal closure that improves drought tolerance. Plant Biotechnology Journal. 2018;17:118-131.

4. Barik S, Sarkardas S, Singh A, Gautam V, Kumar P, Majee M, Sarkar AK. Phylogenetic analysis reveals conservation and diversification of micro RNA166 genes among diverse plant species. Genomics. 2014;103:114-121.

5. Boualem A, Laporte P, Jovanovic M, Laffont C, Plet J, Combier J, Niebel A, Crespi M, Frugier F. MicroRNA166 controls root and nodule development in Medicago truncatula. The Plant Journal. 2008;54:876-887.

6. Bushati N, Cohen SM. MicroRNA functions. Annu Rev Cell Dev Biol. 2007;23:175-205.

7. Carlsbecker A, Lee J, Roberts C, Dettmer J, Lehesranta S, Zhou J, Lindgren O, Morenorisueno MA, Vaten A, Thitamadee S, Campiho A, Sebastian J, Bowman JL, Helariutta Y, Benfey PN. Cell signaling by microRNA165/6 directs gene dose-dependent root cell fate. Nature. 2010;465:316-321.

8. Cassanwang H, Goue N, Saidi MN, Legay S, Sivadon P, Goffner D, Grimapettenati J. Identification of novel transcription factors regulating secondary cell wall formation in Arabidopsis. Frontiers in Plant Science. 2013;4:189.

9. Chen XM. A microRNA as a translational repressor of APETALA2 in Arabidopsis flower development. Science. 2004;303:2022-2025.

10. Côté CL, Boileau F, Roy V, Ocuellet M, Levasseur C, Morency MJ, Cooke JEK, Seguin A, Mackay JJ. Gene family structure, expression and functional analysis of HD-Zip III genes in angiosperm and gymnosperm forest trees. BMC Plant Biol. 2010;10:273.

11. Cuperus JT, Fahlgren N, Carrington JC. Evolution and functional diversification of MIRNA genes. Plant Cell. 2011;23:431-442.

12. Depuydt S, Hardtke CS. Hormone signaling crosstalk in plant growth regulation. Curr Biol. 2011;21:365-373.

13. Du Q, Avci U, Li SB, Gallegogiraldo L, Pattathil S, Qi LY, Hahn MG, Wang HZ. Activation of miR165b represses AtHB15 expression and induces pith secondary wall development in Arabidopsis. The Plant Journal. 2015;83:388-400.

14. Ferdous J, Hussain SS, Shi BJ. Role of microRNAs in plant drought tolerance. Plant Biotechnology Journal. 2015;13:293-305.

15. Floyd SK, Bowman JL. Ancient microRNA regulation of gene expression in land plants. Nature. 2004;428:485-486.

16. Floyd SK, Zalewski CS, Bowman JL. Evolution of class III homeodomain-leucine zipper genes in streptophytes. Genetics. 2006;173:373-388.

17. Han J, Fang JG, Wang C, Yin YL, Sun X, Leng XP, Song CN. Grapevine microRNAs responsive to exogenous gibberellin. BMC Genomics. 2014;15:111.

18. Itoh J, Hibara K, Sato Y, Nagato Y. Developmental role and auxin responsiveness of Class III homeodomain leucine zipper gene family members in rice. Plant Physiol. 2008;147:1960-1975.

19. Jonesrhoades MW, Bartal D. Computational identification of plant microRNAs and their targets, including a stress-induced miRNA. Mol Cell. 2004;14:787-799.

20. Jung J, Park C. MIR166/165 genes exhibit dynamic expression patterns in regulating shoot apical meristem and floral development in Arabidopsis. Planta. 2007;225:1327-1338.

21. Kim J, Jung J, Reyes JL, Kim Y, Kim S, Chung K, Kim JA, Lee M, Lee Y, Kim VN, Chua N, Park C. microRNA-directed cleavage of ATHB15 mRNA regulates vascular development in Arabidopsis inflorescence stems. The Plant journal. 2005;42:84-94.

22. Kim Y, Kim S, Lee M, Lee L, Park H, Seo PJ, Jung J, Kwon E, Suh SW, Paek KH, Park C. HD-ZIP III activity is modulated by competitive inhibitors via a feedback loop in Arabidopsis shoot apical meristem development. Plant Cell. 2008;20:920-933.

23. Kinoshita N, Wang H, Kasahara H, Liu J, Macpherson CR, Machida Y, Kamiya Y, Hannah MA, Chua N. IAA-Ala resistant3, an evolutionarily conserved target of miR167, mediates Arabidopsis root architecture changes during high osmotic stress. Plant Cell. 2012;24:3590-3602.

24. Ko JH, Prassinos C, Han KH. Developmental and seasonal expression of PtaHB1, a Populus gene encoding a class III HD-Zip protein, is closely associated with secondary growth and inversely correlated with the level of microRNA (miR166). New Phytologist. 2006;169:469-478.

25. Leung AKL, Sharp PA. MicroRNA functions in stress responses. Mol Cell. 2010;40:205-215.

26. Li AL, Mao L. Evolution of plant microRNA gene families. Cell Research. 2007;17:212-218.

27. Li XY, Xie X, Li J, Cui YH, Hou YM, Zhai LL, Wng X, Fu YL, Liu RR, Bian SM. Conservation and diversification of the miR166 family in soybean and potential roles of newly identified miR166s. BMC Plant Biology. 2017;17:32.

28. Miyashima S, Honda M, Hashimoto K, Tatematsu K, Hashimoto T, Satonara K, Okada K, Nakajima K. A comprehensive expression analysis of the arabidopsis MICRORNA165/6 gene family during embryogenesis reveals a conserved role in Meristem specification and a non-cell-autonomous function. Plant Cell Physiol. 2013;54:375-384.

29. Miyashima S, Koi S, Hashimoto T, Nakajima K. Non-cell-autonomous microRNA165 acts in a dose-dependent manner to regulate multiple differentiation status in the Arabidopsis root. Development. 2011;138:2303-2313.

30. Ohashiito K, Kubo M, Demura T, Fukuda H. Class III homeodomain leucine-zipper proteins regulate xylem cell differentiation. Plant Cell Physiol. 2005;46:1646-1656.

Page 11/19 
31. Palatnik JF, Allen E, Wu XL, Schommer C, Schwab R, Carrington JC, Weigel D. Control of leaf morphogenesis by microRNAs. Nature. 2003;425:257263.

32. Singh A, Roy S, Singh S, Das SS, Gautam V, Yadav S, Kumar A, Singh A, Samantha S, Sarkar AK. Phytohormonal crosstalk modulates the expression of miR166/165s, target Class III HD-ZIPS, and KANADI genes during root growth in Arabidopsis thaliana.Scientific Reports. $2017 ; 7: 3408$.

33. Singh A, Singh S, Panigrahi KCS, Reski R, Sarkar AK. Balanced activity of microRNA166/165 and its target transcripts from the class III homeodomain-leucine zipper family regulates root growth in Arabidopsis thaliana. Plant Cell Reports. 2014;33:945-953.

34. Song XW, Li Y, Cao XF, Qi YJ. MicroRNAs and Their Regulatory Roles in Plant-Environment Interactions. Annual Review of Plant Biology. 2019;70:489-525.

35. Taku D, Fukuda H. Transcriptional regulation in wood formation. Trends in Plant Science. 2007;12:64-70.

36. Tang MY, Bai X, Niu LJ, Chai X, Chen MS, Xu ZF. MiR172 Regulates both Vegetative and Reproductive Development in the Perennial Woody Plant Jatropha curcas. Plant and Cell Physiology. 2018;59:2549-2563.

37. Wang AH, Hu JH, Gao CB, Chen GL, Wang BC, Lin CF, Song LP, Ding Y, Zhou GL. Genome-wide analysis of long non-coding RNAs unveils the regulatory roles in the heat tolerance of Chinese cabbage (Brassica rapa ssp.chinensis). Scientific Reports. 2019;9:5002.

38. Wang C, Han J, Liu CH, Kibet KN, Kayesh E, Shangguan LF, Li XY, Fang JG. Identification of microRNAs from Amur grape (Vitis amurensis Rupr.) by deep sequencing and analysis of microRNA variations with bioinformatics. BMC Genomics. 2012;13:122.

39. Wang C, Jogaiah S, Zhang WY, Abdelrahman M, Fang JG. Spatio-temporal expression of miRNA159 family members and their GAMYB target gene during the modulation of gibberellin-induced grapevine parthenocarpy. Journal of Experimental Botany. 2018;69:3639-3650.

40. Wang JW, Czech B, Weigel D. MiR156-Regulated SPL Transcription Factors Define an Endogenous Flowering Pathway in Arabidopsis thaliana. Cell. 2009;138:738-749.

41. Wang WJ, Qi YJ. Plant non-coding RNAs and epigenetics. Science China Life Sciences. 2018;61:135-137.

42. Wang WR, Bai YH, Koilkonda P, Guan L, Zhuge YX, Wang XC, Liu ZJ, Jia HF, Wang C, Fang JG. Genome-wide identification and characterization of gibberellin metabolic and signal transduction (GA MST) pathway mediating seed and berry development (SBD) in grape (Vitis vinifera L.). BMC Plant Biol. 2020;20:384.

43. Wang WR, Wang C, Xie ZQ, Jia HF, Tang W, Cui MJ, Fang JG. Function Analysis of VvmiR397a and Its Target Genes VvLACs in Grape Berry Development. Acta Horticulturae Sinica. 2018,45(08):1441-1455.

44. Wenkel S, Emery J, Hou B, Evans MMS, Barton MK. A feedback regulatory module formed by LITTLE ZIPPER and HD-ZIPIII genes. Plant Cell. 2007;19:3379-3390.

45. Williams L, Grigg SP, Xie MT, Christensen S, Fletcher JC. Regulation of Arabidopsis shoot apical meristem and lateral organ formation by microRNA miR166g and its AtHD-ZIP target genes. Development. 2005;132:3657-3668.

46. Wu G, Park MY, Conway SR, Wang JW, Weigel D, Poethig RS. The sequential action of miR156 and miR172 regulates developmental timing in Arabidopsis. Cell. 2009;138:750-759.

47. Zhang J, Xie M, Tuskan GA, Muchero W, Chen JG. Recent Advances in the Transcriptional Regulation of Secondary Cell Wall Biosynthesis in the Woody Plants. Frontiers in Plant Science. 2018;9:1535.

48. Zhang QQ, Wang JG, Wang LY, Wang JF, Wang Q, Yu P, Bai MY, Fan M. Gibberellin repression of axillary bud formation in Arabidopsis by modulation of DELLA-SPL9 complex activity. Journal of Intergrative Plant Biology. 2019;Doi:10.1111/jipb.12818.

49. Zhang WY, Abdelrahman M, Jiu ST,Guan L, Han J, Zheng T, Jia HF, Song CN, Fang JG, Wang C. VvmiR160s/VvARFs interaction and their spatiotemporal expression/cleavage products during GA-induced grape parthenocarpy. BMCPlant Biol. 2019,21(19):111.

50. Zhang XH, Zou Z, Zhang JH, Zhang YY, Han QQ, Hu TX, Xu XG, Liu H, Li HX, Ye ZB. Over-expression of sly-miR156a in tomato results in multiple vegetative and reproductive trait alterations and partial phenocopy of the sft mutant. Febs Letters. 2011; 585:435-439.

\section{Tables}

Table1 Effect of GA on fruit quality of 'Bailuosha'

\begin{tabular}{|c|c|c|c|c|c|c|c|c|c|}
\hline GA & A Period & & B Period & & C Period & & D Period & & \\
\hline $\begin{array}{l}\text { concentration } \\
(\mathrm{mg} / \mathrm{L})\end{array}$ & $\begin{array}{l}\text { single fruit } \\
\text { Weight }(g)\end{array}$ & $\begin{array}{l}\text { Fruit } \\
\text { shape } \\
\text { index }\end{array}$ & $\begin{array}{l}\text { single fruit } \\
\text { Weight }(\mathrm{g})\end{array}$ & $\begin{array}{l}\text { Fruit } \\
\text { shape } \\
\text { index }\end{array}$ & $\begin{array}{l}\text { single fruit } \\
\text { Weight }(\mathrm{g})\end{array}$ & $\begin{array}{l}\text { Fruit } \\
\text { shape } \\
\text { index }\end{array}$ & $\begin{array}{l}\text { single fruit } \\
\text { Weight }(\mathrm{g})\end{array}$ & $\begin{array}{l}\text { Fruit } \\
\text { shape } \\
\text { index }\end{array}$ & $\begin{array}{l}\text { Seedless } \\
\text { rate }(\%)\end{array}$ \\
\hline 50 & $2.755 a$ & $1.66 a$ & $3.89 a$ & $1.4 a$ & $5.26 a$ & $1.49 a$ & $6.61 a$ & $1.65 a$ & $\mathrm{Ob}$ \\
\hline 0 & $2.63 a$ & $1.4 \mathrm{~b}$ & $3.79 a$ & $1.08 b$ & $5.115 a$ & $1.08 b$ & $6.41 a$ & $1.22 \mathrm{~b}$ & $96 a$ \\
\hline
\end{tabular}

Note: $a$ and $\mathrm{b}$ indicate significant difference at 0.05 level ,A period:32days after floweringB period:46 days after flowering, $\mathrm{C}$ period: 60 days after flowering,D period:80 days after flowering 


\begin{tabular}{|c|c|c|c|c|c|c|}
\hline miRNA_Acc & miRNA_Length & Expectation & UPE & Target_Acc. & Target_Regions & Inhibition \\
\hline \multirow[t]{16}{*}{ TCGGACCAGGCGTTCATTCC } & 20 & 3 & 22.742 & VIT_204s0008g03250.1DHB-15] & $\begin{array}{l}\text { CDS:1205- } \\
1223\end{array}$ & Cleavage \\
\hline & 20 & 3 & 22.742 & VIT_204s0008g03250.2వHB-15! & $\begin{array}{l}\text { CDS:1205- } \\
1223\end{array}$ & Cleavage \\
\hline & 20 & 3 & 21.088 & VIT_210s0003g04670.1HOX32] & $\begin{array}{l}\text { CDS:1065- } \\
1083\end{array}$ & Cleavage \\
\hline & 20 & 3 & 21.088 & VIT_210s0003g04670.2[HОХ32】 & CDS:431-449 & Cleavage \\
\hline & 20 & 3 & 23.076 & VIT_210s0003g04670.3[НОХ32] & $\begin{array}{l}\text { CDS:1159- } \\
1177\end{array}$ & Cleavage \\
\hline & 20 & 3 & 23.995 & $\begin{array}{l}\text { VIT_213s0019g04320.10REVOLUTA- } \\
\text { like』] }\end{array}$ & $\begin{array}{l}\text { CDS:1114- } \\
1132\end{array}$ & Cleavage \\
\hline & 20 & 3 & 23.995 & $\begin{array}{l}\text { VIT_213s0019g04320.2』REVOLUTA- } \\
\text { like』 }\end{array}$ & $\begin{array}{l}\text { CDS:1114- } \\
1132\end{array}$ & Cleavage \\
\hline & 20 & 3 & 23.995 & $\begin{array}{l}\text { VIT_213s0019g04320.3ロREVOLUTA- } \\
\text { like[ }\end{array}$ & $\begin{array}{l}\text { CDS:1114- } \\
1132\end{array}$ & Cleavage \\
\hline & 20 & 3 & 23.995 & $\begin{array}{l}\text { VIT_213s0019g04320.4』REVOLUTA- } \\
\text { like] }\end{array}$ & CDS:444-462 & Cleavage \\
\hline & 20 & 3 & 23.995 & $\begin{array}{l}\text { VIT_213s0019g04320.5[REVOLUTA- } \\
\text { like』 }\end{array}$ & CDS:387-405 & Cleavage \\
\hline & 20 & 3 & 23.995 & $\begin{array}{l}\text { VIT_213s0019g04320.6ロREVOLUTA- } \\
\text { like[ }\end{array}$ & $\begin{array}{l}\text { CDS:1114- } \\
1132\end{array}$ & Cleavage \\
\hline & 20 & 3 & 23.995 & $\begin{array}{l}\text { VIT_213s0019g04320.7ロREVOLUTA- } \\
\text { like[ }\end{array}$ & $\begin{array}{l}\text { CDS:1114- } \\
1132\end{array}$ & Cleavage \\
\hline & 20 & 3 & 22.12 & VIT_206s0004g02800.1ロREVOLUTA] & $\begin{array}{l}\text { CDS:1322- } \\
1340\end{array}$ & Cleavage \\
\hline & 20 & 3 & 22.12 & VIT_206s0004g02800.2\REVOLUTAD & $\begin{array}{l}\text { CDS: } 1730- \\
1748\end{array}$ & Cleavage \\
\hline & 20 & 3 & 22.12 & VIT_206s0004g02800.3[REVOLUTAD & $\begin{array}{l}\text { CDS:1746- } \\
1764\end{array}$ & Cleavage \\
\hline & 20 & 3 & 18.711 & VIT_203s0097g00550.1 & CDS:919-938 & Translation \\
\hline \multirow[t]{14}{*}{ TCGGACCAGGCTTCATTCCCC } & 21 & 2 & 22.742 & VIT_204s0008g03250.1[HB-15] & $\begin{array}{l}\text { CDS:1204- } \\
1223\end{array}$ & Cleavage \\
\hline & 21 & 2 & 22.742 & VIT_204s0008go3250.2[HB-15] & $\begin{array}{l}\text { CDS:1204- } \\
1223\end{array}$ & Cleavage \\
\hline & 21 & 2 & 21.088 & VIT_210s0003g04670.1ロHOX32] & $\begin{array}{l}\text { CDS:1064- } \\
1083\end{array}$ & Cleavage \\
\hline & 21 & 2 & 21.088 & VIT_210s0003g04670.2ロHOX32】 & CDS:430-449 & Cleavage \\
\hline & 21 & 2 & 23.076 & VIT_210s0003g04670.3[НОХ32] & $\begin{array}{l}\text { CDS:1158- } \\
1177\end{array}$ & Cleavage \\
\hline & 21 & 2 & 23.995 & $\begin{array}{l}\text { VIT_213s0019g04320.1ロREVOLUTA- } \\
\text { like』 }\end{array}$ & $\begin{array}{l}\text { CDS:1113- } \\
1132\end{array}$ & Cleavage \\
\hline & 21 & 2 & 23.995 & $\begin{array}{l}\text { VIT_213s0019g04320.2』REVOLUTA- } \\
\text { like] }\end{array}$ & $\begin{array}{l}\text { CDS:1113- } \\
1132\end{array}$ & Cleavage \\
\hline & 21 & 2 & 23.995 & $\begin{array}{l}\text { VIT_213s0019g04320.3[REVOLUTA- } \\
\text { like[] }\end{array}$ & $\begin{array}{l}\text { CDS:1113- } \\
1132\end{array}$ & Cleavage \\
\hline & 21 & 2 & 23.995 & $\begin{array}{l}\text { VIT_213s0019g04320.4』REVOLUTA- } \\
\text { like[ }\end{array}$ & CDS:443-462 & Cleavage \\
\hline & 21 & 2 & 23.995 & $\begin{array}{l}\text { VIT_213s0019g04320.5』REVOLUTA- } \\
\text { like』 }\end{array}$ & CDS:386-405 & Cleavage \\
\hline & 21 & 2 & 23.995 & $\begin{array}{l}\text { VIT_213s0019g04320.6ロREVOLUTA- } \\
\text { like』 }\end{array}$ & $\begin{array}{l}\text { CDS:1113- } \\
1132\end{array}$ & Cleavage \\
\hline & 21 & 2 & 23.995 & $\begin{array}{l}\text { VIT_213s0019g04320.7DREVOLUTA- } \\
\text { like[ }\end{array}$ & $\begin{array}{l}\text { CDS:1113- } \\
1132\end{array}$ & Cleavage \\
\hline & 21 & 2 & 22.12 & VIT_206s0004g02800.1[REVOLUTAD & CDS:1321- & Cleavage \\
\hline & & & Page & & & \\
\hline
\end{tabular}


1340

\begin{tabular}{|c|c|c|c|c|c|}
\hline 21 & 2 & 22.12 & VIT_206s0004g02800.2 2REVOLUTA & $\begin{array}{l}\text { CDS:1729- } \\
1748\end{array}$ & Cleavage \\
\hline 21 & 2 & 22.12 & VIT_206s0004g02800.3[REVOLUTA] & $\begin{array}{l}\text { CDS:1745- } \\
1764\end{array}$ & Cleavage \\
\hline 21 & 2.5 & 20.995 & VIT_218s0075g00480.1 & $\begin{array}{l}\text { CDS:3094- } \\
3113\end{array}$ & Translation \\
\hline 21 & 2.5 & 20.995 & VIT_218s0075g00480.2 & $\begin{array}{l}\text { CDS:3094- } \\
3113\end{array}$ & Translation \\
\hline 21 & 2.5 & 20.995 & VIT_218s0075g00480.3 & $\begin{array}{l}\text { CDS:2850- } \\
2869\end{array}$ & Translation \\
\hline 21 & 2.5 & 20.995 & VIT_218s0075g00480.4 & $\begin{array}{l}\text { CDS:2830- } \\
2849\end{array}$ & Translation \\
\hline 21 & 2.5 & 20.995 & VIT_218s0075g00480.5 & $\begin{array}{l}\text { CDS:3094- } \\
3113\end{array}$ & Translation \\
\hline 21 & 2.5 & 20.995 & VIT_218s0075g00480.6 & $\begin{array}{l}\text { CDS:3327- } \\
3346\end{array}$ & Translation \\
\hline 21 & 2.5 & 20.995 & VIT_218s0075g00480.7 & $\begin{array}{l}\text { CDS:2066- } \\
2085\end{array}$ & Translation \\
\hline 21 & 3 & 18.46 & VIT_216s0050g01140.1 & CDS:357-376 & Translation \\
\hline 21 & 3 & 17.375 & VIT_208s0007g03420.1 & CDS:364-383 & Cleavage \\
\hline
\end{tabular}

Note: The sequences of miRNAs and their target genes were compared by BioXM software to predict the mismatch numbers and the target regions.

Table 3 Functional annotation of predicted target genes

\begin{tabular}{|c|c|c|c|c|c|}
\hline Target gene & & Interval & length & $\begin{array}{l}\mathrm{E}- \\
\text { value }\end{array}$ & Target gene annotation \\
\hline VIT_206s0004g02800 & REVOLUTA & $\begin{array}{l}8460- \\
8621\end{array}$ & 161 & $\begin{array}{l}6.51 \mathrm{E}- \\
13\end{array}$ & homeobox leucine-zipper protein \\
\hline VIT_213s0019g04320 & $\begin{array}{l}\text { REVOLUTA- } \\
\text { like }\end{array}$ & $\begin{array}{l}2456- \\
2617\end{array}$ & 161 & $\begin{array}{l}6.04 \mathrm{E}- \\
13\end{array}$ & homeobox-leucine zipper protein revoluta \\
\hline VIT_204s0008g03250 & $H B-15$ & $\begin{array}{l}8733- \\
8897\end{array}$ & 164 & $\begin{array}{l}1.61 \mathrm{E}- \\
12\end{array}$ & $\begin{array}{l}\text { class iii hd-zip protein } 8 \text { homeobox-leucine zipper protein athb-15 } \\
\text { hypothetical protein }\end{array}$ \\
\hline VIT_210s0003g04670 & HOX32 & $380-541$ & 161 & $\begin{array}{l}1.56 \mathrm{E}- \\
13\end{array}$ & homeobox-leucine zipper protein hox32-like \\
\hline
\end{tabular}

Note: The Grapevine Genome CRIBI Biotech website (http://genomes.cribi.unipd.it/grape/search_form.php) was used to identify the target gene name, grape ID, coordinates and theirfunctions.

Table 4 Sequences of primer

\begin{tabular}{lll} 
Genes primers & Forward primer sequences $\left(5^{\prime}-3^{\prime}\right)$ & Reverse primer sequences $\left(5^{\prime}-3^{\prime}\right)$ \\
\hline 166 a qRT-PCR & AGGAAAGCTTGGTGTCAGGA & GGAAAGCGGGTGTTTGGAAT \\
\hline $166 \mathrm{~b}$ qRT-PCR & TTTGAGGGGATGTTGGCTG & CCTAGATGGCCGTGACTGAA \\
\hline $166 \mathrm{c} \mathrm{qRT-PCR}$ & AGATCAAGCCCGAGAAAAGC & TCTGGCTCGAGGACACTTAC \\
\hline $166 \mathrm{~d}$ qRT-PCR & CGATTGGGGAGCAGTGATTG & GAGGCCACCAACCATCTTTG \\
\hline $166 \mathrm{e}$ qRT-PCR & AATGTTGTCTGGCTCGAGGA & GATCAGCAGCAATGGAAGCA \\
\hline $166 f$ qRT-PCR & GTTGTGCTGTGTGTGGTGTA & AAGAAGAGGAGGAGGAGGGT \\
\hline $166 \mathrm{~g}$ qRT-PCR & TCCGGCATGCAAATTGAAGT & GGGGAATGTTGTCTGGTTCG \\
\hline $166 \mathrm{~h}$ qRT-PCR & GGAAGGTTGAGAAGTTGAGCC & ATCTCGGACCAGGCTTCATT \\
\hline HOX32 qRT-PCR & TTACAGAAGCAGGTCTCGCA & GGTTTTGCTGTTGTTGGTGC \\
\hline ATHB-15 qRT-PCR & CTCTGCTCTGAGTTCCCACA & AGCATTGTCCTCTTCGGTCA \\
\hline REVOLUTAqRT-PCR & AGGGTTTTGCTTACTTGCCG & AAGCAAGGCAATGGACAGTG
\end{tabular}

Page 14/19 
Figures

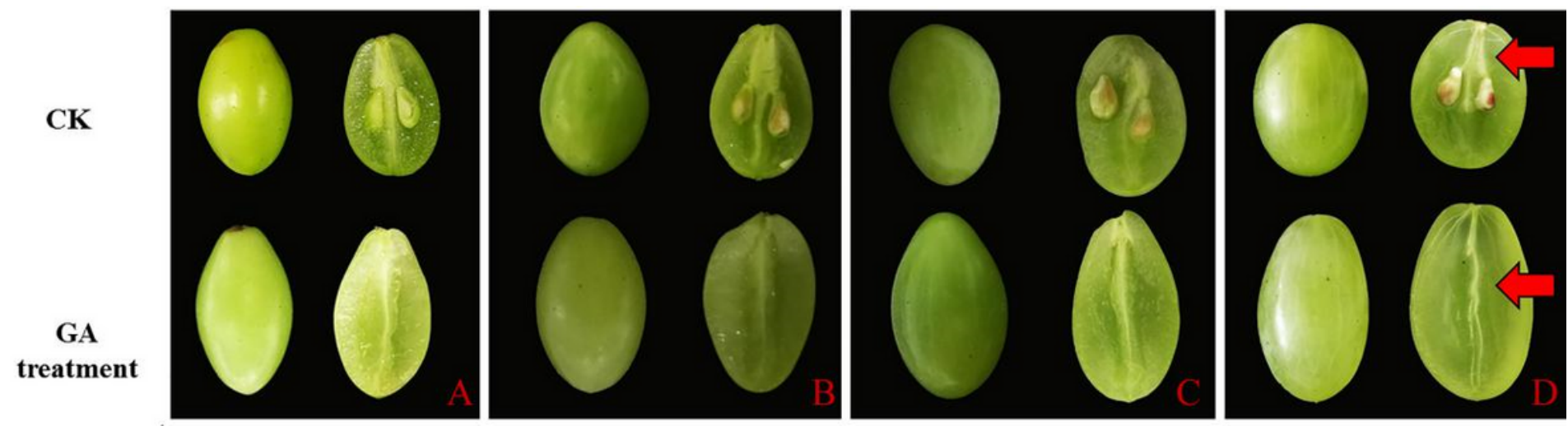

Figure 1

Morphological changes in berries and seeds in response to GA application

A. berries and seeds from untreated and GA treatment plants $32 \mathrm{~d}$ after flowering; B. berries and seeds from untreated and GA treatment plants $46 \mathrm{~d}$ after flowering; C. berries and seeds from untreated and GA treatment plants $60 \mathrm{~d}$ after flowering; $\mathrm{D}$. berries and seeds from untreated and GA treatment plants $86 \mathrm{~d}$ after flowering $\bigotimes$ Red arrow points to fruit brush.

\section{Figure 2}

Comparison of VvmiR166s and their precursor sequences with the alignment, respectively

A. Comparison of VvmiR166s sequence in miRbase 21.0 and cloned VvmiR166s sequence, the red regions are different sequences, the yellow regionsare the same sequences; $B$. Comparison of $V V, M I R 166 a-b$ andVVMIR166c- $h$ sequences in miRbase 21.0, respectively, the red box regions represent the mature sequences of VvmiR166s.

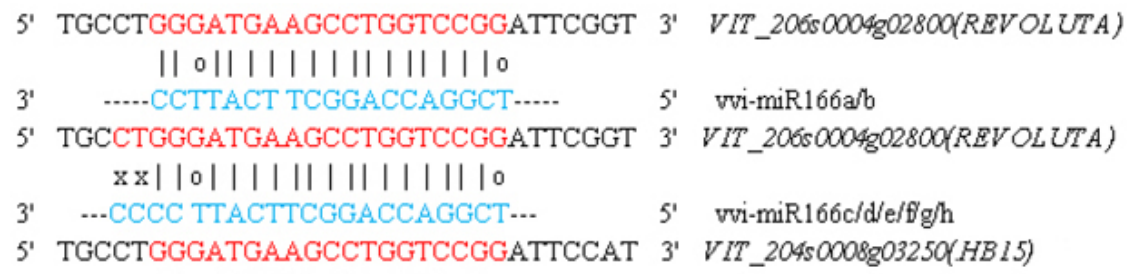

\section{Figure 3}

The complementary degree of VvmiR166s and their potential targets 
Comparison of VvmiR166s sequence in miRbase 2.1.0 and their target genes, the mismatch bases of VvmiR166s with their target genes, ' $X$ ' represents completely mismatch, and ' $O$ ' represents the 0.5 mismatch.
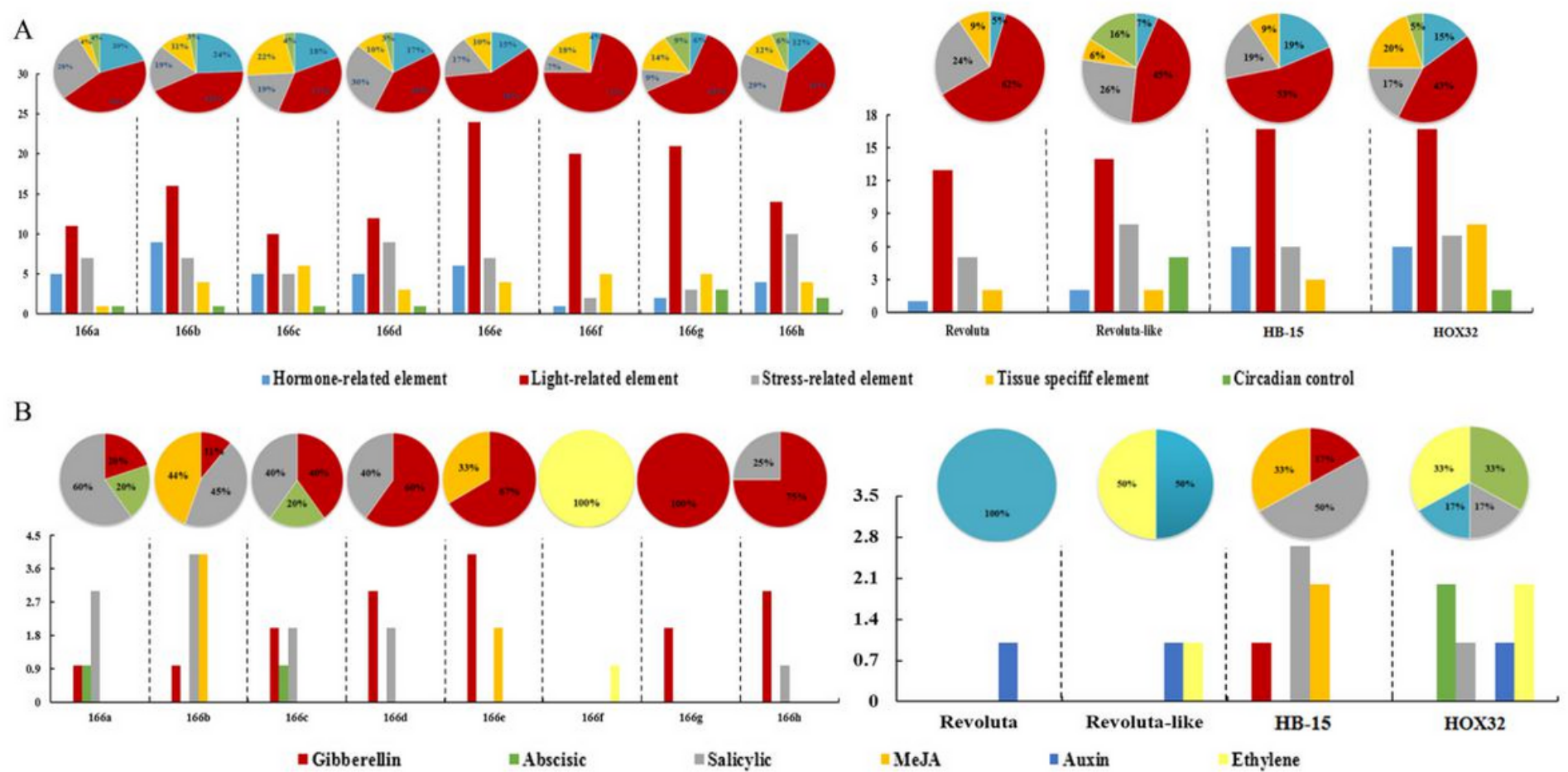

Figure 4

Motif analysis of promoters of VvMIR166s and their potential targets

A. Diverse types of motifs. All the elements are divided into five types: light-related elements; hormone-related elements; stress responsive elements; tissue-specific elements and circadian-related elements. The different position of the diamonds on the histogram picture represents the various number of each type of elements. The pie chart represents the proportion of motif.

B. Various kinds of hormones related to motifs. All the hormone-related elements are divided into six types: Gibberellin related elements; Abscisicacidrelated elements; Salicylic acid-related elements; Methyl jasmonate-related elements;Auxin-related elements; Ethylene-related elements. The different position of the diamonds on the histogram picture represents the various number of each type of elements. The pie chart represents the proportion of motif.

\section{Figure 5}

Phylogenetic tree of $H B 15, H O X 32$ and REV across various plant species

1: Homeodomain domain (HD); 2: Leucine-Zipper domain (Zip); 3: Steroidogenic Acute Regulatory Protein-Related Lipid Transfer domain (START); MEKHLA domain. VvHOX32: Vitis vinifera, XM_002281832; PpHB-14: Prunus persica, XM_007214560; PtPHABULOSA: Populus trichocarpa, XM_002298856; FvHB-14: Fragaria vesca, XM_004293941; OsHOX32: Oryza sativa, XM_015776998; NtHOX32: Nicotiana tabacum, XM_009614046; NtREVOLUTA: Nicotiana tabacum, NM_001325575; AtREVOLUTA: Arabidopsis thaliana, NM_125462; VvREVOLUTA: Vitis vinifera, XM_010652862; FvREVOUTA: Fragaria vesca, XM_004291525; PpREVOLUTA: Prunus persica, XM_007208028; AtHB-15: Arabidopsis thaliana: NM_104096; VvHB-15: Vitis vinifera, XM_002283681; PpHB-8: Prunus persica, XM_007225219; PtHB-15: Populus trichocarpa, NC_008472; FvHB-8: Fragaria vesca, NC_020492; LeHB-15: lycopersicum esculentum, NC_015445; NtHB-15: Nicotiana tabacum, NW_015945691.

\section{Figure 6}

Subcellular localization of $V_{V} H B 15, V_{v} H O X 32$ and $V v R E V$ in agroinfiltrated tobacco leaves.

Subcellular localization of $V v H B 15, V_{v} H O X 32, V_{V} R E V$, and GFP-fused fluorescent protein transient expression in tobacco leaves. 


\section{Figure 7}

Mapping the cleavage sites of VvmiR166s on their target genes

Bottom strand (red) depicts a miRNA complementary site, and top strand depicts the mRNA (black). Watson-Crick pairing (vertical dashes) and G:U wobble pairing (circles and X) are indicated. The arrows indicate the cleavage sites of VvmiR166s on Target genes. The numbers denote the cleavage frequency of VvmiR166s on Target genes at the cleavage sites. Percentage of the diverse cleavage sites of VvmiR166s on their target genes. Analysis of the cleavage sites of the various VvmiR166s on the same targeted genes

\section{Figure 8}

Expression profiles of VVMIR166s in the various tissues of grape berriesatdiverse stages

32DAF: 32 days after flowering, 46DAF: 46 days after flowering, 60DAF: 60days after flowering, 86DAF:86 days after flowering.Each reaction was repeated three times and standard error was pointed with bars in the diagram.

REVOLUTA

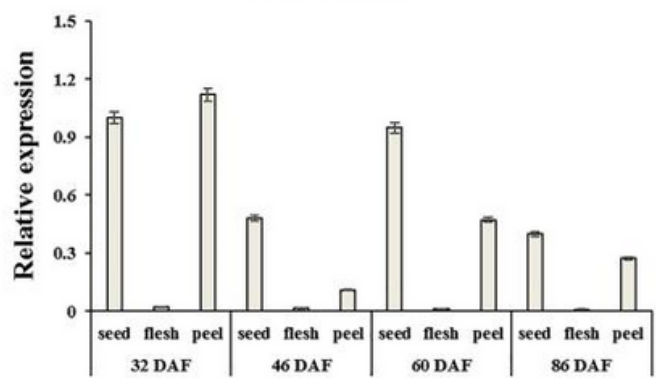

HB-15

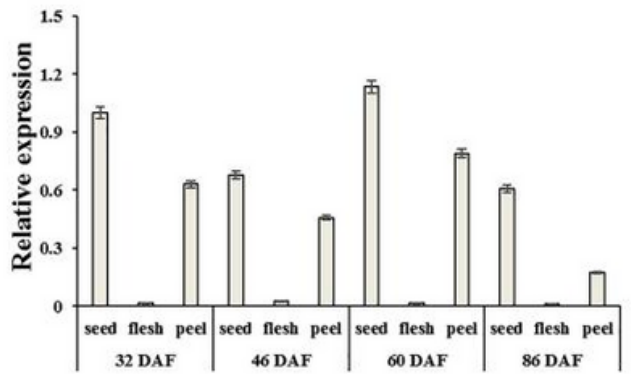

HOX32

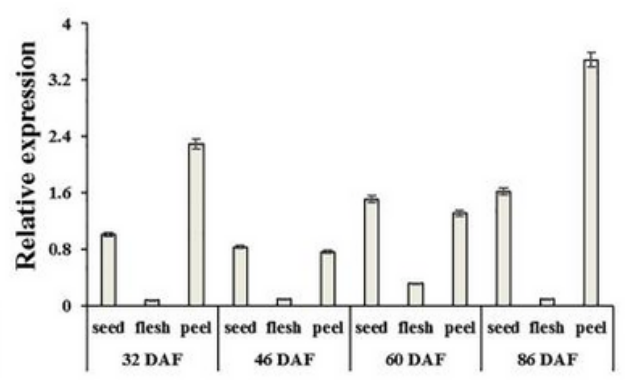

\section{Figure 9}

Expression profiles of the target mRNAs for VvmiR166s in the various tissues of grape berries at diverse stages

32DAF:32 days after flowering, 46DAF:46 days after flowering, 60DAF:60 days after flowering, 86DAF:86 days after flowering.Each reaction was repeated three times and standard error was pointed with bars in the diagram. 
Flesh-HB-15
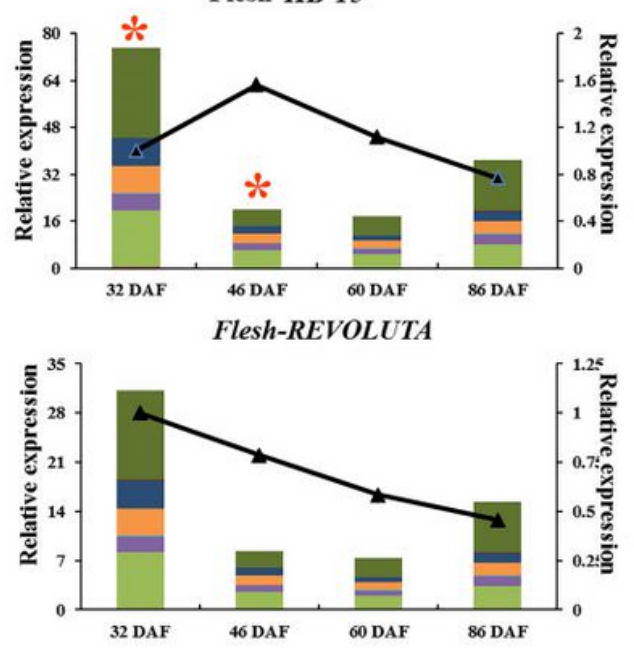

Flesh-HOX32

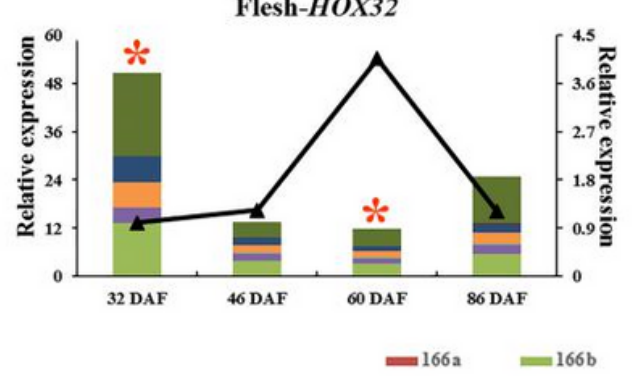

Peel- $\mathrm{HB}-15$
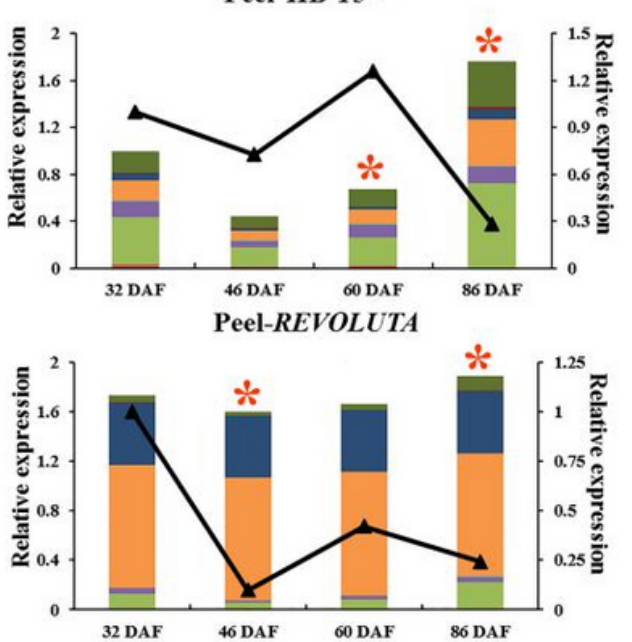

Peel-HOX32

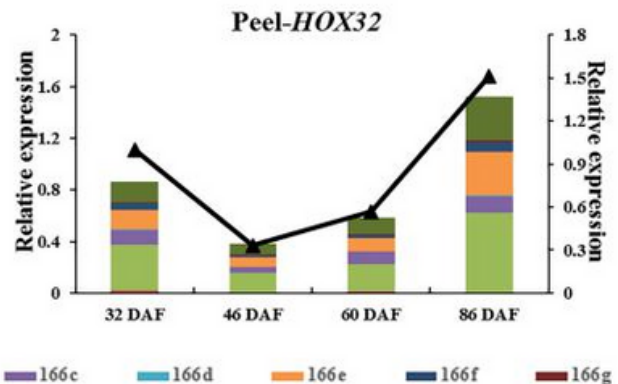

Seed-HB-15
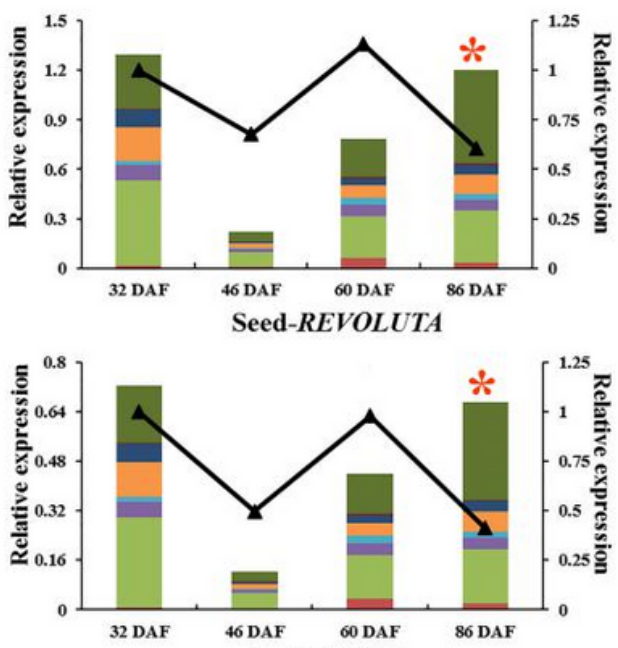

Seed-HOX32

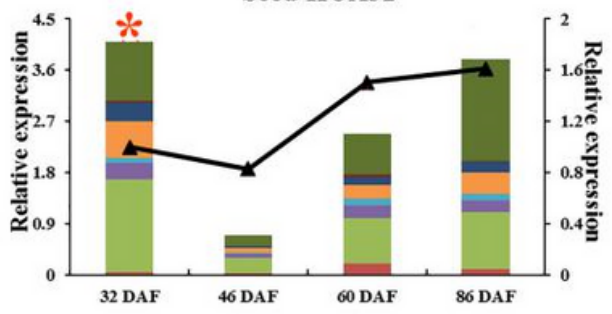

$=166 \mathrm{~h} \quad-$ Target gene

Figure 10

Correlation of total VvMIR166s and their targets in expression levels

The column chart shows the accumulation of the relative expression of each precursor gene in the VvmiR166sfamily,the line chart shows the relative expression of the target gene in different tissues.* marked indicates the opposite expression pattern32DAF:32 days after flowering, 46DAF:46 days after flowering, 60DAF:60 days after flowering, 86DAF:86 days after flowering. Each reaction was repeated three times and standard error was pointed with bars in the diagram.

Figure 11

Expression modes of VVMIR166s responsive to GA in various tissues of grape berries at diverse stages

The column chart shows the relative expression of the untreated tissue,and the line chart shows the relative expression of theGA treatment tissue.32DAF:32 days after flowering, 46DAF:46 days after flowering, 60DAF:60 days after flowering, 86DAF:86 days after flowering.Each reaction was repeated three times and standard error was pointed with bars in diagram.

\section{Figure 12}

Expression modes of $V V A T H B 15, V_{V} R E V$, and $V V H O X 32$ responsive to GA in various tissues of grape berries at diverse stages

The column chart shows the relative expression of the untreated tissue,and the line chart shows the relative expression of theGA treatment tissue.32DAF:32 days after flowering, 46DAF:46 days after flowering, 60DAF:60 days after flowering, 86DAF:86 days after flowering.Each reaction was repeated three times and standard error was pointed with bars in the diagram.

\section{Figure 13}

Comparison of pearson correlation of VvmiR166s and $V v H B 15$ expression between controls and GA treatments 
This chart mainly focused on comparison of expression correlation of VvmiR166s and $V v H B 15$ from control and GA treatments at the two typical phases including the stone hardening stage (from 32DAF to 46DAF) and turning color and maturation stage (from 60DAF to 86DAF); the blue $※$ denotes the comparison in controls, while the red $※$ indicates those in GA treatments.

\section{Figure 14}

Transient expression verification of $V v M I R 166$ sand their target gene $V v H B 15$ in tobacco leaves

A. VvmiR166a-h and $V v H B 15$ were integrated into the plant expression vector pCAMBIA1302 by double digestion.

B. The obtained vector was independently transformed into tobacco plants using an Agrobacterium-mediated method. The expression vector pCAMBIA1302 was used as control. After 3 days of dark culture, DNA was extracted, and specific primers were used for PCR detection.After gel electrophoresis, sequencing was performed, which was identical to the sequence of interest.

C. Agrobacterium-mediated injection of tobacco leaves.

D. After overexpressing VvmiR166a-h and $V V A T H B$, the expression changes of genes involved in tobacco lignin synthesis pathway were detected by real-time quantitative PCR.'a, b, c, d, e, f, g, h' and 'at' indicate overexpression of VvmiRa-h and $V v H B 15$, respectively. 'ck' is the control.

E. After overexpressing VvmiR166a-h and $V v H B 15$, the expression changes of NtHB1 and NtHB2.

\section{Figure 15}

-VvmiR166b/h-VvHB15- lignin synthesis genes pathway

The chart comprise of two parts, of which one part is the development processes of grape berries, especially seeds, in controls(CK) and GA treatments, in which A, B, C, D denotes the four stages of 32, 46, 60, 86 days after flowering, while another part is the network of GA-mediated VvmiR166s and their targets in lignin synthesis, and the arrows represent the promotion, and the hammer denote the repression.

\section{Supplementary Files}

This is a list of supplementary files associated with this preprint. Click to download.

- SupplementarydataTableS1.docx

- SupplementarydataTables2.docx 